\title{
Descarte de medicamentos: uma análise da prática de usuários de uma farmácia pública no noroeste do Rio Grande do Sul
}

\section{Discard of medicines: an analysis of the practice of users of a public pharmacy in the northwest of Rio Grande do Sul}

Desecho de medicamentos: análisis de la práctica de usuarios de una farmacia pública en el noroeste de Rio Grande do Sul

Fernanda Andressa Sheer ${ }^{1}$ Regis Augusto Norbert Deuschle ${ }^{2}$ Gabriela Bonfanti Azzolin ${ }^{3}$ Viviane Cecilia Kesser Nunes Deuschle ${ }^{4}$

${ }^{1}$ Graduada em Farmácia pela Universidade de Cruz Alta (Unicruz). E-mail: nandaascheer@hotmail.com, Orcid: http://orcid.org/0000-0001-8343-8582.

Unicruz, Cruz Alta, Rio Grande do Sul, Brasil.

${ }^{2}$ Graduado em Farmácia pela Universidade Federal de Santa Maria (UFSM). Pró-reitor de Graduação e docente do Curso de Farmácia da Universidade de Cruz Alta.E-mail: rdeuschle@unicruz.edu.br, Orcid: http://orcid.org/0000-0001-6527-4449

${ }^{3}$ Graduada em Farmácia pela Universidade Federal de Santa Maria (UFSM). Docente do Curso de Farmácia e do Mestrado em Atenção Integral à Saúde na Universidade de Cruz Alta (Unicruz). E-mail: gbonfanti@unicruz.edu.br, Orcid: http://orcid.org/0000-0003-2602-6092

${ }^{4}$ Graduada em Farmácia pela Universidade de Cruz Alta (Unicruz). Docente dos Cursos de Farmácia e Tecnologia em Estética e Cosmética da Unicruz. E-mail: vdeuschle@unicruz.edu.br, Orcid: http://orcid.org/0000-0001-6797-0376 
Resumo: Este estudo descreveu as práticas de uso e descarte de medicamentos entre usuários de uma Farmácia Pública na região noroeste do Estado do Rio Grande do Sul. A determinação foi realizada mediante aplicação de um questionário e a amostra foi constituída por 80 participantes. Os dados levantados demonstram que a maioria dos entrevistados $(96,25 \%)$ apresenta medicamentos em suas residências e 65\% relataram praticar a automedicação. Os resultados também demonstram que 38,75\% dos participantes descartam os medicamentos no lixo seco doméstico. Em relação às informações sobre as formas adequadas de descartes, 52,5\% já receberam algum tipo de informação, mas $81,25 \%$ gostariam de receber mais informações sobre o assunto. Portanto observa-se que grande parcela da população estudada descarta os medicamentos de forma incorreta e ainda carece de informação quanto à forma de descarte, demonstrando uma necessidade de serviços de educação em saúde no sistema público estudado.

Palavras-chave: automedicação; riscos para a saúde; preservação ambiental; educação em saúde; uso racional de medicamentos.

\begin{abstract}
This study described practices of use and disposal of drugs among users of a Public Pharmacy in the northwest region of the State of Rio Grande do Sul. The determination was performed by means of a questionnaire and the sample consisted of 80 participants. The data showed that most of the interviewees (96.25\%) had drugs in their homes and 65\% reported practicing self-medication. The results also show that $38.75 \%$ of the participants discard the drugs in the domestic dry garbage. Regarding information on the appropriate forms of discarding, $52.5 \%$ already received some type of information, but $81.25 \%$ would like to receive more information on the subject. Therefore, it is observed that a large part of the studied population incorrectly discards medicines and still lacks information on the way of disposal, demonstrating a need for health education services in the public system studied.
\end{abstract}

Keywords: self-medication; health risks; environmental preservation; health education; rational drug use.

Resumen: Este estudio describió las prácticas de uso y eliminación de medicamentos entre los usuarios de una farmacia pública en la región noroeste del estado de Rio Grande do Sul. La determinación se realizó mediante un cuestionario y la muestra estuvo compuesta por 80 participantes. Los datos mostraron que la mayoría de los entrevistados (96,25\%) tenían drogas en sus hogares y el $65 \%$ reportó haber practicado automedicación. Los resultados también muestran que el 38,75\% de los participantes descarta los medicamentos en la basura doméstica. Con respecto a la información sobre las formas apropiadas de descarte, el 52,5\% ya recibió algún tipo de información, pero el 81,25\% desea recibir más información sobre el tema. Por lo tanto, se observa que una gran parte de la población estudiada desecha incorrectamente los medicamentos y aún carece de información sobre el modo de eliminación, lo que demuestra la necesidad de servicios de educación sanitaria en el sistema público estudiado.

Palabras clave: la automedicación; riesgos para la salud; preservación del medio ambiente; educación en salud; uso racional de medicamentos. 
Descarte de medicamentos: uma análise da prática de usuários de uma farmácia pública no noroeste do Rio Grande do Sul

\section{INTRODUÇÃO}

Segundo a Constituição Federal Brasileira de 1988, a educação e conscientização para preservar o meio ambiente são deveres do Estado, com a intenção de proteger e diminuir os danos causados pelo homem à natureza.

Algo que causa um grande impacto no meio ambiente são as substâncias químicas usadas diariamente em indústrias e, principalmente, as relacionadas à área da saúde. Os medicamentos contêm substâncias químicas (fármacos e outros excipientes) que fazem parte do cotidiano de muitas famílias, especialmente quando há doentes crônicos, pois são formulados para tratar ou amenizar sintomas de patologias ou disfunções, devendo ser usados continuamente.

Atualmente, com a produção em larga escala, o acesso a esses produtos se dá como o de qualquer outro tipo de comércio, isto é, não há uso racional. Detecta-se uma falta de conhecimento, tanto para os usuários e sua família como também para alguns prescritores e pessoas que dispensam medicamentos, e um consequente acúmulo de medicamento nas residências. Ainda, boa parte dos pacientes é polimedicada, favorecendo o acúmulo de vários tipos de medicamentos que, com o passar do tempo, perdem sua estabilidade e, assim, sua eficácia, principalmente por terem a data de validade expirada. Também há os antimicrobianos provenientes de tratamentos incompletos, sobras de tratamentos de pessoas que faleceram, hormônios que o paciente não se adaptou, entre outros. Todos esses produtos que fazem parte da chamada "farmácia caseira" precisam ser descartados em algum momento.

A Resolução 358/2005, do Conselho Nacional do Meio Ambiente (CONAMA), que dispõe sobre a preservação dos recursos naturais do meio ambiente, e a RDC 306/2004, da Agência Nacional de Vigilância Sanitária (ANVISA), integram o controle dos processos de gerenciamento dos resíduos, incluindo todo o seu manejo. Esses documentos auxiliam os estabelecimentos de saúde que precisam dar a disposição final dos resíduos, a qual é normalmente feita através de terceirização, por empresas específicas, mediante contrato de prestação de serviço. Entretanto, apesar da alta taxa de dispensação de medicamentos, as Farmácias Públicas Municipais não são obrigadas a prestar esse serviço à população. Assim, o descarte doméstico 
continua sendo feito em grande quantidade e de maneira inadequada, uma vez que há dificuldade por parte da população em encontrar locais adequados para dar o destino correto aos seus medicamentos.

A população, sem informação sobre o uso racional e consumo sustentável, acaba descartando em locais comuns os medicamentos que sobram ou que não serão mais utilizados, como o lixo orgânico e reciclável, vaso sanitário, ralo da pia ou diretamente no ambiente. Essas substâncias são absorvidas e contaminam o solo e as águas e, mesmo com a realização do tratamento do esgoto, muitas vezes não são neutralizadas ou eliminadas completamente, causando danos para toda a fauna e flora e colocando em risco a saúde da população.

No Brasil, os programas de coleta de medicamentos vencidos ou fora de uso visam reduzir os riscos de intoxicações, acidentes e a automedicação, promovendo o uso racional de medicamentos, além de contribuir para a redução dos riscos ambientais.

Neste contexto, o objetivo deste trabalho é investigar práticas de uso e descarte de medicamentos de usuários de uma Farmácia Pública, assim como o nível de conhecimento em relação ao descarte correto e ao impacto ambiental e para a saúde.

\section{METODOLOGIA}

A presente investigação caracteriza-se como sendo observacional transversal, analítica e descritiva, com abordagem quantitativa, o que caracteriza o estudo de campo.

Os participantes da pesquisa foram os usuários de uma Farmácia Pública localizada no município de Panambi, região noroeste do Estado do Rio Grande do Sul, que possui, em média, 40.000 habitantes. A Farmácia Pública atende um número entre 200 e 300 pessoas diariamente. Os dados foram coletados nos meses de agosto e setembro de 2018, quando foram entrevistados 80 voluntários, os quais responderam a questões abertas e fechadas para a obtenção dos dados referentes à forma como os seus medicamentos vencidos ou fora de uso são descartados. A seleção dos entrevistados ocorreu de acordo com a disponibilidade em responder 
durante sua espera ao atendimento e, para participar da pesquisa, os voluntários deveriam frequentar a farmácia pública no período da aplicação do questionário, ter idade mínima de 18 anos e compreender o objetivo de sua participação. As pessoas que não se enquadraram nesses quesitos não participaram do estudo.

Esta pesquisa respeitou os aspectos éticos em relação à pesquisa com seres humanos, de acordo com a Resolução 466/2012, do Conselho Nacional de Saúde (BRASIL, 2012). Foi emitida uma Carta de Autorização ao Responsável pela Farmácia Pública antes do início da pesquisa. Este trabalho foi previamente aprovado pelo Comitê de Ética em Pesquisa (CEP) da Universidade de Cruz Alta, sob o parecer consubstanciado número 2.605.901. Foram entrevistados somente os que concordaram em participar de maneira voluntária, assinando o Termo de Consentimento Livre e Esclarecido (TCLE). Os pesquisadores assinaram o Termo de Confidencialidade, comprometendo-se com o sigilo dos dados coletados.

Para representação das variávies categóricas, foi utilizada frequência relativa e absoluta e, para as variáveis quantitativas, média e desvio padrão (DP).

\section{RESULTADOS E DISCUSSÃO}

O público que frequenta a Farmácia Pública do município de Panambi é bastante variado em relação a sua faixa etária. A faixa etária dos participantes do estudo foi em média de $46 \pm 16$ anos de idade. Tal variação ocorre pelas inúmeras classes de medicamentos disponíveis aos usuários do Sistema Único de Saúde (SUS), atendendo doenças e complicações decorrentes de todas as idades. Apenas 27,5\% corresponderam ao gênero masculino e $72,5 \%$ dos participantes pertenciam ao gênero feminino. Esse resultado superior para as mulheres pode ser devido ao fato de possuírem maior expectativa de vida e também por se preocuparem mais com os cuidados da sua saúde. Além disso, normalmente, elas se responsabilizam mais pela saúde de sua família, assumindo esse papel (BUENO; WEBER; OLIVEIRA, 2009; RUPPENTHAL; PETROVICK, 2010).

Com relação ao nível de escolaridade, constatou-se que 33,75\% dos voluntários possuem o ensino médio completo, 27,5\% possuem ensino 
fundamental incompleto, apenas 1,25\% não são alfabetizados e 13,73\% possuem ensino superior. Já na pesquisa de Bueno, Weber e Oliveira (2009), realizada num bairro no município de ljuí, RS, o número de voluntários não alfabetizados chegou a $10 \%$; $61 \%$ possuíam ensino fundamental incompleto; quase $7 \%$ completaram o ensino médio; e apenas 0,62\% possuía ensino superior completo. Esses dados demonstram que, de alguma forma, a população está buscando cada vez mais por conhecimento, de acordo com suas possibilidades. Além disso, o nível de estudo individual é relacionado com a renda pessoal, mostrando que a renda tende a crescer de acordo com o aumento dos anos de estudo (RUPPENTHAL; PETROVICK, 2010).

Sobre os aspectos relacionados com a forma de armazenamento dos medicamentos nas residências dos participantes, a aparência deles, a presença de doenças e a prática da automedicação, os dados coletados encontram-se sumarizados na Tabela 1.

Tabela 1 - Práticas de uso e descarte de medicamentos

\begin{tabular}{|c|c|c|}
\hline \multirow{2}{*}{ QUESTÕES } & \multicolumn{2}{|c|}{$\begin{array}{l}\text { RESPOSTAS } \\
(\%)\end{array}$} \\
\hline & SIM & NÃO \\
\hline Possui medicamentos em casa? & 96,25 & 3,75 \\
\hline $\begin{array}{l}\text { Os medicamentos estão ao alcance das crianças? (caso façam } \\
\text { parte do convívio na residência) }\end{array}$ & 3,75 & 96,25 \\
\hline Observa o aspecto/aparência do medicamento antes de utilizá-lo? & 83,75 & 16,25 \\
\hline $\begin{array}{l}\text { Possui insumos necessários à prestação de primeiros socorros na } \\
\text { farmácia caseira, tais como algodão, gaze, termômetro, } \\
\text { esparadrapo e antissépticos? }\end{array}$ & 66,25 & 26,25 \\
\hline Possui medicamentos vencidos? & 6,25 & 93,75 \\
\hline $\begin{array}{l}\text { Já recebeu alguma informação quanto ao descarte de } \\
\text { medicamentos que você não usa mais? }\end{array}$ & 52,5 & 47,5 \\
\hline $\begin{array}{l}\text { Gostaria de ter mais informações sobre a maneira correta de } \\
\text { descartar os medicamentos e sobre as consequências de descartar } \\
\text { incorretamente? }\end{array}$ & 81,25 & 18,75 \\
\hline $\begin{array}{l}\text { Quando você ou alguém em sua casa está doente, vocês praticama } \\
\text { automedicação? }\end{array}$ & 65,0 & 35,0 \\
\hline
\end{tabular}

Fonte: Os autores. 
Descarte de medicamentos: uma análise da prática de usuários de uma farmácia pública no noroeste do Rio Grande do Sul

Ao serem questionados sobre a presença de medicamentos em casa, 96,25\% responderam "sim". Pereira, Paula e Santos (2018) entrevistaram 300 famílias, atendidas em uma Unidade Básica de Saúde do município de Bocaiúva, MG, e verificaram que $82 \%$ dos participantes possuíam medicamentos em casa. Já Ferreira, Santos e Rodrigues (2015) entrevistaram 386 pessoas na cidade de Belo Horizonte, MG, com o objetivo de avaliar o conhecimento da população sobre o descarte de medicamentos e os impactos ambientais causados por ações incorretas, e constataram que 95\% dos participantes possuíam medicamentos.

Mastroianni et al. (2011) realizaram um estudo em São Paulo no intuito de descrever a ocorrência de estoque, armazenamento e modo de uso de medicamentos em 280 domicílios atendidos por uma Estratégia Saúde da Família (ESF) e descreveram que em 91\% das residências foram encontrados estoques de medicamentos.

Ao serem questionados sobre observarem o aspecto/aparência do medicamento antes da utilização (Tabela 1), 83,75\% dos participantes responderam afirmativamente. Silva, Abjaude e Rascado (2014) obtiveram $70 \%$ de respostas positivas. Sabe-se que o medicamento, mesmo estando no prazo de validade, pode sofrer alterações, principalmente se for armazenado de maneira incorreta ou exposto a atritos muito fortes durante seu transporte. Desta forma, é de extrema importância a verificação de seu aspecto antes da administração, uma vez que se podem evitar danos à saúde ou ao comprometimento da terapia (PINTO et al., 2016).

Os voluntários que afirmaram possuir insumos para primeiros socorros atingiram 66,25\% (Tabela 1), muito semelhante ao resultado de Bueno, Weber e Oliveira (2009), que somou 63,25\%. Os dados demonstram que há uma preocupação da população em ter esses itens em casa para suprir casos de emergência, mas esta não supera a necessidade de possuírem medicamentos domiciliares (BUENO; WEBER; OLIVEIRA, 2009; MARIANI; OLIVEIRA, 2015).

Em nosso estudo, 96,25\% dos participantes responderam que os medicamentos não estão ao alcance de crianças (Tabela 1). Mariani e Oliveira (2015) obtiveram um resultado de 95,55\%, em que os participantes relatam ter este mesmo cuidado, demonstrando uma preocupação com os riscos de intoxicações medicamentosas em crianças, decorrentes de ingestão 
acidental. Contudo Bueno, Weber e Oliveira (2009) demonstraram, em seu estudo, que $30 \%$ dos participantes afirmaram que os medicamentos são armazenados em locais de fácil acesso de crianças, condizendo com os resultados obtidos por Lima, Nunes e Barros (2010), em que 54\% relataram que os medicamentos ficam ao alcance delas.

Segundo dados do Sistema Nacional de Informações Tóxico-Farmacológicas (SINITOX), em 2016 (BRASIL, 2016), as intoxicações decorrentes de medicamentos em crianças de um a quatro anos atingiram $27,69 \%$ dos casos. As principais formas de intoxicação ocorrem por via oral (76\%), com pequena participação de outras vias, como tópica e inalatória (6\%). Na idade de um a quatro anos, as formas farmacêuticas sólidas, como cápsulas e comprimidos, são as responsáveis por 44,9\% das intoxicações. Crianças são potenciais usuários de medicamentos devido à facilidade com que contraem doenças e essa prática, muitas vezes, estimula a utilização acidental ou por brincadeiras (MAIOR; OLIVEIRA, 2012).

Os fatores que levam ao aumento de intoxicações nessa faixa etária incluem as diferenças farmacocinéticas e farmacodinâmicas em relação aos adultos, uma vez que os medicamentos são calculados em relação ao peso. Todavia deve-se levar em consideração também a área corporal, a idade, a porcentagem de gordura, proteínas e água - desta forma, a dose administrada nem sempre garantirá a segurança e eficácia do tratamento em relação a sua fase de desenvolvimento (MAIOR; OLIVEIRA, 2012; SILVA; OLIVEIRA, 2018).

Em relação à presença de medicamentos vencidos em casa, 93,75\% relataram não os ter, mas, no estudo de Silva e Geron (2018), foram encontrados medicamentos vencidos em $11 \%$ das residências que participaram da pesquisa. Ainda, em um estudo realizado por Mastroianni et al. (2011), esse resultado foi bastante expressivo, uma vez que foram encontrados medicamentos com o prazo de validade vencido em 42,3\% dos domicílios dos participantes. Neste caso, a pesquisa realizada entre a população cadastrada em uma ESF da cidade de São Paulo detectou problemas de segurança e identificação dos medicamentos armazenados, o que pode caracterizar um risco à saúde.

Entre os participantes de nosso estudo, 52,5\% relataram que já receberam algum tipo de informação sobre o descarte de medicamentos, 
Descarte de medicamentos: uma análise da prática de usuários de uma farmácia pública no noroeste do Rio Grande do Sul

significando que pouco menos da metade da amostra nunca recebeu tais informações.

Ferreira, Santos e Rodrigues (2015) avaliaram o conhecimento da população sobre o descarte de resíduos através de entrevistas com 386 pessoas em Belo Horizonte, MG, e verificaram que $87 \%$ dos participantes não receberam informações, o que demonstra um descuido dos profissionais da área da saúde com um assunto de extrema importância e que pode trazer muitos danos ao meio ambiente e à população que desconhece a maneira correta de descartar os medicamentos.

Todavia, $81,25 \%$ dos participantes de nossa pesquisa gostariam de receber mais informações a respeito do descarte correto de medicamentos. Quando há interesse da população em entender melhor os assuntos que envolvem toda a comunidade, atividades como campanhas de incentivo e educação em saúde se tornam muito mais produtivas e geram um impacto importante na sociedade. Essas atividades são de responsabilidade, principalmente, do Estado e estão descritas na Constituição, porém qualquer pessoa que já possui entendimento em relação ao assunto também tem o dever de transmitir este conhecimento, de maneira a contribuir para o melhoramento da comunidade, tanto em aspectos culturais como sociais (FERREIRA; SANTOS; RODRIGUES, 2015; GASPARINI; GASPARINI; FRIGIERI, 2011; PINTO et al., 2016).

Como 96,25\% dos participantes disseram possuir medicamentos em suas residências, foram questionados também sobre a prática da automedicação, pois é uma atividade bastante difundida no Brasil. Sobre essa questão, 65\% afirmaram praticar a automedicação. Em um estudo desenvolvido por Silva e Geron (2018), em que foram avaliados o armazenamento e a quantidade dos medicamentos vencidos em 410 domicílios, eles observaram que $67 \%$ dos participantes se automedicavam. No estudo de Aquino, Barros e Silva (2010), realizado com uma amostra de 223 estudantes da área da saúde, 57,7\% relataram que praticam a automedicação. Contudo, no estudo de Bueno, Weber e Oliveira (2009) realizado em ljuí, RS, os quais verificaram a prática da farmácia caseira e o descarte de medicamentos através da aplicação de um questionário que alcançou 321 entrevistados, o número elevou-se a $75,7 \%$. 
A automedicação é um assunto que muito preocupa os profissionais da saúde, pois não há uso responsável na maioria das vezes. Além disso, pode prejudicar algum tratamento médico que se encontra em andamento e causar intoxicações ou outros problemas de saúde se for administrado por via ou dose incorreta (PINTO; LUSTOSA; FERNANDES, 2017).

Em seguida, os voluntários responderam quais as indicações dos medicamentos armazenados em casa (tabela 2), sendo que, nesta questão, era possível assinalar mais de uma alternativa.

Tabela 2 - Indicações terapêuticas de fármacos armazenados nas residências

\begin{tabular}{l|c}
\hline \multicolumn{1}{c|}{ Para quais indicações? } & $\%$ \\
\hline Dor & $\mathbf{7 7 , 5 0}$ \\
Febre & $\mathbf{5 0 , 0 0}$ \\
Doenças crônicas (diabetes, asma, pressão & $\mathbf{5 8 , 7 5}$ \\
alta, entre outras) & $\mathbf{3 8 . 7 5}$ \\
Antialérgicos & $\mathbf{2 0 , 0 0}$ \\
Antibióticos & $\mathbf{4 3 , 7 5}$ \\
Anti-inflamatórios & \\
\hline
\end{tabular}

Fonte: Os autores.

A maioria dos entrevistados $(77,5 \%)$ informou possuir medicamentos para dor. Resultados semelhantes foram encontrados no estudo de Aquino, Barros e Silva (2010), em que eles observaram que também foi a classe mais utilizada (24\%), talvez pela facilidade na compra, uma vez que a maioria destes são medicamentos isentos de prescrição (MIPs) e possuem elevado índice de automedicação (AQUINO; BARROS; SILVA, 2010; BUENO; WEBER; OLIVEIRA, 2009).

Ainda, nossos resultados apontam a presença de anti-inflamatórios nas residências, que chegaram a 43,75\%; antitérmicos, 50\% (principalmente entre os participantes que possuíam crianças em casa); antialérgicos, 
Descarte de medicamentos: uma análise da prática de usuários de uma farmácia pública no noroeste do Rio Grande do Sul

38,75\%; e 20\% possuíam antibióticos ou alguém da família estava fazendo uso naquele momento. Mais da metade dos participantes $(58,75 \%)$ tinham medicamentos indicados para uma ou mais doenças crônicas.

Em um estudo realizado por Ruppernthal e Petrovick (2010) em Porto Alegre, com o objetivo de comparar perfis de usuários da Farmácia Popular do Brasil da UFRGS e de um estabelecimento privado de dispensação a respeito das características de medicamentos quanto à classe terapêutica, quantidade dispensada e uso crônico, verificou-se que 65\% de todos os produtos dispensados eram usados para o sistema cardiovascular.

No estudo de Bueno, Weber e Oliveira (2009), 41,11\% das 321 pessoas entrevistadas eram acometidas por hipertensão arterial sistêmica (HAS). Já em outro estudo, de Mariani e Oliveira (2015), dos 100 indivíduos entrevistados, 33\% possuíam alguma doença crônica. As doenças crônicas levam o paciente a utilizar medicamentos diariamente e, portanto, sempre há presença de medicamentos em casa. Entretanto, em algumas situações, quando não é feito o tratamento adequadamente, pode haver acúmulo desnecessário ou expirar o prazo de validade (MARIANI; OLIVEIRA, 2015; RUPPERNTHAL; PETROVICK, 2010).

O armazenamento dos medicamentos é um ponto crítico, pois é de extrema importância manter em local adequado para que não perca sua estabilidade e eficácia e não traga riscos ao usuário (SILVA; GERON, 2018).

De acordo com a Figura 1, observa-se que 30\% dos participantes armazenam seus medicamentos na cozinha. Pereira, Paula e Santos (2018) constataram que $45 \%$ dos entrevistados armazenam os medicamentos na cozinha e no banheiro e Lenhardt et al. (2014) verificaram que 40,5\% armazenam na cozinha.

Segundo Mastroianni et al. (2011), a maioria dos participantes de seu estudo armazenava os medicamentos em local inseguro e inadequado, ou seja, expostos ao sol, umidade, calor, sujeira, animais domésticos ou em presença de substâncias como perfumes, cosméticos e desinfetantes, comprometendo a qualidade e estabilidade do medicamento. Em nosso estudo, $68,75 \%$ armazenam em outros cômodos, como quarto, sala de estar e corredor. Não se pode classificar a adequabilidade desse armazenamento sem mais informações, mas é importante manter em local fresco, arejado e limpo. 
Figura 1 - Local de armazenamento dos medicamentos

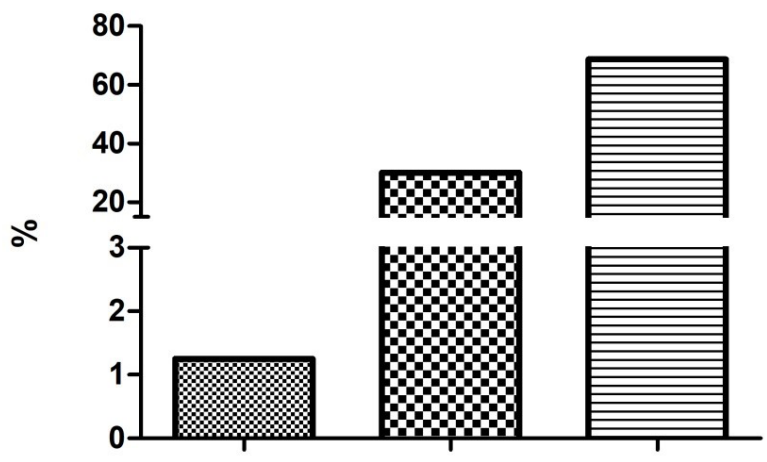

Banheiro $\mathbb{B}$ Cozinha $\square$ Outros cômodos*

*Quarto, corredor, sala de estar.

Fonte: Os autores.

Sobre o destino dado em relação às sobras de medicamentos vencidos ou fora de uso, os resultados encontram-se demonstrados na Figura 2.

Figura 2 - Destino dado às sobras de medicamentos domiciliares

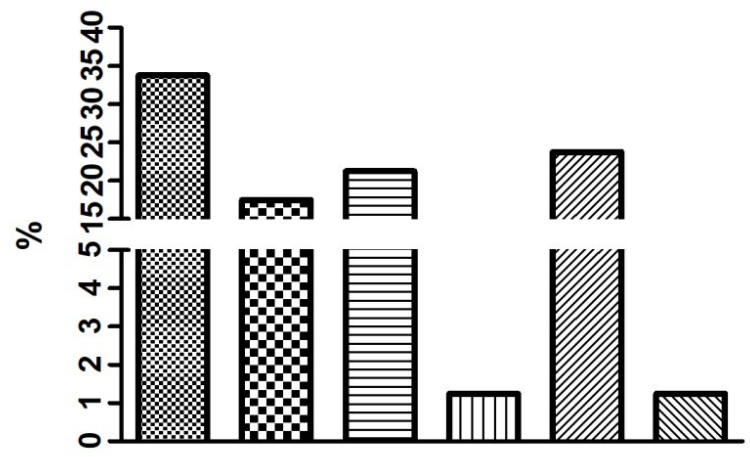

Agente de saúde $\mathbf{B}$ Guarda para usar outra vez

D Descarta

四 Dá aos vizinhos/amigos

שão sobra

Outros

Fonte: Os autores. 
Descarte de medicamentos: uma análise da prática de usuários de uma farmácia pública no noroeste do Rio Grande do Sul

Nesta figura, os resultados demonstram que $17,5 \%$ dos participantes guardam os medicamentos para serem usados novamente. Segundo Mastroianni et al. (2011), o uso de medicamentos vencidos ou sobras de outros tratamentos pode trazer riscos à saúde, pois, além de intoxicações, os medicamentos podem causar efeitos indesejados ou até mesmo não ser efetivo para alguns casos, retardando a cura ou mascarando os sintomas.

Contudo, segundo a Tabela 1, apenas 6,25\% afirmaram possuir medicamentos fora do prazo de validade em casa. No estudo de Bueno, Weber e Oliveira (2009), foi constatado que apenas 5,3\% mantêm medicamentos vencidos e fazem revisões com frequência em suas "farmácias caseiras". 0 número foi um pouco mais elevado no estudo de Silva e Geron (2018), em que $11 \%$ dos domicílios participantes continham medicamentos vencidos.

Segundo a Agência Nacional de Vigilância Sanitária (ANVISA), o prazo de validade de um medicamento corresponde ao "tempo durante o qual o produto poderá ser usado, caracterizado como o período de vida útil fundamentado nos estudos de estabilidade específicos" e, por isso, esse prazo deve ser respeitado (OLIVEIRA et al., 2015).

A realização de tratamentos incompletos, reações adversas a medicamentos e especialidades farmacêuticas com maior número de medicamentos do que o tratamento prescrito podem gerar sobras. Associada a isso, a facilidade de compra de medicamentos nas farmácias pode levar à automedicação. Em outros estudos, como de Gasparini, Gasparini e Frigieri (2011) e Oliveira et al. (2015), 30\% e 31\% dos entrevistados, respectivamente, guardavam as sobras de medicamentos para serem usadas novamente, equivalendo a um número mais elevado ao encontrado em nosso estudo.

Na Figura 3, estão representados os resultados da questão sobre o motivo do descarte dos medicamentos. No total, $77,5 \%$ disseram que os descartam quando estão vencidos, demonstrando que as pessoas tentam não manter os medicamentos fora do prazo de validade na sua "farmácia caseira", e 31,25\% descartam quando não fazem mais uso.

É difícil ocorrer alterações nas características físicas e químicas de um medicamento ainda válido se este for armazenado corretamente, mas manter medicamentos que não serão usados constitui-se em um risco para 
a saúde, aumentando as chances de automedicação (ALBANAZ et al., 2017; AQUINO; BARROS, SILVA, 2010).

Figura 3 - Motivo do descarte dos medicamentos

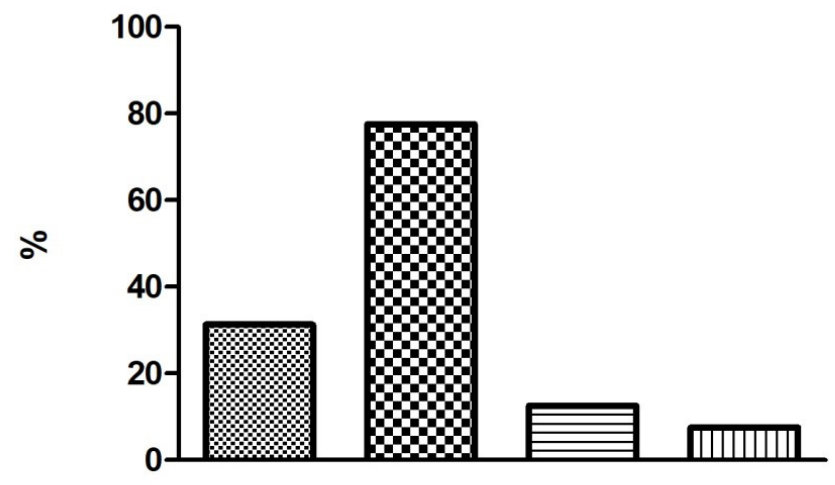

\section{Não usa mais $\square$ Interrompeu o tratamento \\ Estão vencidos $\mathbf{m}$ Aparência estranha}

Fonte: Os autores.

Quando a validade do medicamento é expirada, sua estabilidade fica comprometida e não há motivos para continuar armazenando-o; dessa maneira, deve-se fazer o descarte de forma adequada (SILVA; GERON, 2018).

A Figura 4 resume os dados obtidos em relação à forma como é realizado o descarte dos medicamentos. De acordo com os resultados, $46,5 \%$ relataram entregar em um estabelecimento de saúde e $38,75 \%$ o fazem no lixo seco.

No estudo de Pereira, Paula e Santos (2018), 86\% dos participantes descartam os medicamentos no lixo comum ou no vaso sanitário, $10 \%$ os devolvem à unidade de saúde e apenas $4 \%$ os descartam na farmácia, corroborando com os resultados obtidos em nosso estudo. 
Figura 4 - Local de descarte dos medicamentos

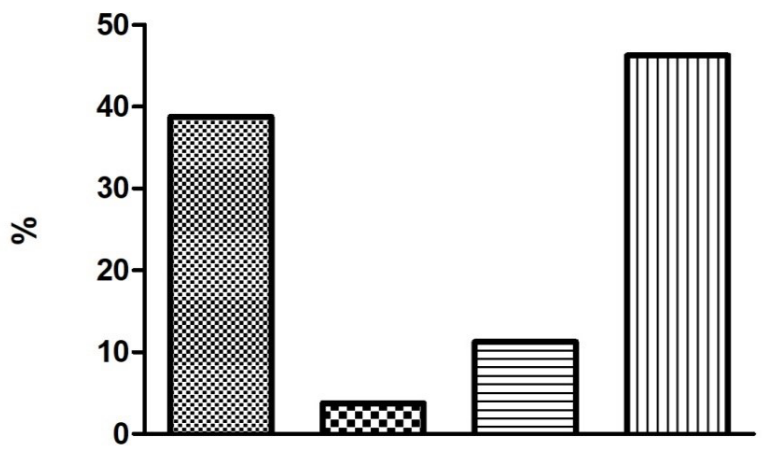

Lixo seco

E Lixo úmido

曰 Vaso sanitário ou pia 而 Entrega em um estabelecimento

Fonte: Os autores.

Ao serem descartadas no lixo comum e levadas aos aterros sanitários, as embalagens dos medicamentos são destruídas e os resíduos de fármacos e excipientes entram em contato com o solo e, assim, podem ser absorvidos ou levados pela água da chuva. Independentemente do caminho que tomar, os produtos químicos vão agindo no meio ambiente. O descarte em vasos sanitários ou pias acarreta em contaminação dos lençóis freáticos (SILVA; GERON, 2018). Além disso, o lixo domiciliar facilita o acesso das crianças e animais domésticos aos fármacos que foram inadequadamente descartados, podendo provocar graves intoxicações (BUENO; WEBER; OLIVEIRA, 2009).

As substâncias farmacologicamente ativas e outros componentes presentes nos medicamentos são substâncias químicas de difícil decomposição e, desta forma, contaminam o meio ambiente. As estações de tratamento de água não conseguem remover essas substâncias, que acabam chegando ao consumidor através da ingestão da água potável. Estudos revelam que já foram encontrados fármacos como cafeína, estradiol, progesterona e etinilestradiol na água potável do município de Campinas, SP (GUERRIERI; HENKES, 2017).

Essas substâncias, quando consumidas, provocam alterações no sistema endócrino, consistindo em riscos para os usuários que não necessitam desses fármacos. Igualmente, pode atingir os animais e provocar alterações 
no sistema sexual, comprometendo a reprodução das espécies (GUERRIERI; HENKES, 2017).

O descarte de medicamentos realizado de maneira inadequada pela maioria das pessoas provém de ausência de informação sobre o assunto (GASPARINI; GASPARINI; FRIGIERI, 2011). Ainda de acordo com a Tabela $1,47,5 \%$ dos entrevistados responderam que ainda não haviam recebido informações quanto ao descarte correto de medicamentos e suas consequências ao meio ambiente. Este porcentual representa um número elevado, pois, nos tempos atuais, a divulgação de notícias, acontecimentos e informações é de fácil acesso à maioria da população. Porém, no estudo de Mariani e Oliveira (2015), 95\% dos participantes revelaram não ter recebido informações quanto ao armazenamento e ao descarte dos medicamentos.

Com isso, destaca-se a relevância desse estudo, uma vez que devem ser intensificadas as discussões acerca do uso racional e consumo sustentável dos medicamentos, descarte adequado das diferentes formas farmacêuticas, impactos ambientais e para a saúde que o descarte incorreto pode acarretar, tanto em instituições de ensino como em estabelecimentos de saúde.

Apesar das farmácias não terem obrigatoriedade de receber os medicamentos fora do prazo de validade ou de uso, torna-se imprescindível estabelecer medidas que aumentem os postos de coleta e o acesso da população, uma vez que são iniciativas importantes no que tange a segurança e saúde dos usuários. Os resultados permitem ainda inferir que, apesar de muitas pessoas terem acesso à informação, faltam opções de descarte em estabelecimentos e unidades de saúde e que mais informações sobre os riscos decorrentes do descarte inadequado devem fazer parte de ações educativas em saúde.

\section{CONCLUSÃO}

O estudo aponta as características desta população em relação ao descarte dos medicamentos, seu uso e seu armazenamento nas residências, visando reduzir os riscos ambientais e para a saúde. Contribuiu para mostrar que, apesar de muitas pessoas terem informações sobre esses riscos, muitas vezes não sabem onde realizar o descarte. Além disso, pode-se 
Descarte de medicamentos: uma análise da prática de usuários de uma farmácia pública no noroeste do Rio Grande do Sul

observar que a automedicação é uma prática frequente entre as pessoas, o que pode colocar a saúde em risco. Neste sentido, ressalta-se a importância de ações educativas sobre o uso racional e descarte adequado de medicamentos fora de uso ou com prazo de validade expirado. Observamse, ainda, as dificuldades em se estabelecerem pontos de recebimento de medicamentos, uma vez que os estabelecimentos de saúde não têm essa obrigatoriedade e, quando o fazem, caracterizam-se como ações sociais em benefícios da comunidade. Com isso, há uma necessidade urgente de se elaborarem normas e estratégias para intensificar as campanhas de uso racional de medicamentos e redução dos estoques de medicamentos domiciliares, diminuindo, assim, a automedicação e o descarte incorreto.

\section{REFERÊNCIAS}

ALBANAZ, Heitor Felipe; PRADO, Jellifer Rodrigues; CRUZ, Renato Araújo; BARBOSA, Andréia Aparecida; BLANCO, Bruno Anjos. Descarte de medicamentos: uma panorâmica da atual situação. Revista Gestão em Foco, Registro, v. 9, p. 276-90, 2017.

AQUINO, Daniela Silva; BARROS, José Augusto Cabral; SILVA, Maria Dolores Paes. A automedicação e os acadêmicos da área da saúde. Ciência \& Saúde Coletiva, Rio de Janeiro, v. 15, n. 5, p. 2533-8, 2010.

BRASIL. Conselho Nacional de Saúde. Portaria n. 466/2012, de outubro de 2012. Dispõe sobre diretrizes e normas regulamentadoras de pesquisa com seres humanos. Diário Oficial da União, Brasília, DF, 13 de junho de 2013, Seção 1, p. 59.

BRASIL. Ministério da Saúde. Sistema Nacional de Informações TóxicoFarmacológicas (SINITOX). Casos de intoxicação por medicamentos por unidade federada, segundo faixa etária registrado em 2016. Brasília-DF, 2016. Disponível em: https://sinitox.icict.fiocruz.br/dados-de-agentes-toxicos. Acesso em: 27 out. 2018.

BUENO, Cristiane Schmalz.; WEBER, Débora; OLIVEIRA, Karla Renata. Farmácia caseira e descarte de medicamentos no bairro Luiz Fogliatto do município de ljuíRS. Revista de Ciências Farmacêuticas Básica e Aplicada, São Paulo, v. 30, n. 2, p. 75-82, 2009.

FERREIRA, Claudiney Luís; SANTOS, Maria Aparecida Souza dos; RODRIGUES, Shirlene Campos. Análise do conhecimento da população sobre descarte de 
medicamentos em Belo Horizonte - Minas Gerais. Interfaces Científicas - Saúde e Ambiente, Aracajú, v. 3, n. 2, p. 9-18, 2015.

GASPARINI, Joice do Carmo; GASPARINI, André Renah; FRIGIERI, Mariana Carina. Estudo do descarte de medicamentos e consciência ambiental no município de Catanduva-SP. Ciência \& Tecnologia, Jaboticabal, v. 2, n. 1, p. 38-51, 2011.

GUERRIERI, Fernanda de Mesquita; HENKES, Jairo Afonso. Análise do descarte de medicamentos vencidos: um estudo de caso no município de Rio das Ostras (RJ). Revista Gestão e Sustentabilidade Ambiental, Florianópolis, v. 6, n. 1, p. 566-608, 2017.

LENHARDT, Elizandra Hertel; SÓLIS, Laynara Jéssica Burg; CINTRA, Elizângela Vicuna Couto da Silva; BOTELHO, Elizabeth Hertel Lenhardt. O Descarte de medicamentos no bairro Grande Terceiro, Cuiabá-MT. Unopar Científica: Ciências Biológicas e da Saúde, Londrina, v. 16, n. 1. p. 5-8, 2014.

LIMA, Geandra Batista; NUNES, Lívio César Cunha; BARROS, José Augusto Cabral. Uso de medicamentos armazenados em domicílio em uma população atendida pelo Programa Saúde da Família. Ciência \& Saúde Coletiva, Rio de Janeiro, v. 15, n. 3, p. 3517-22, 2010.

MAIOR, Marta da Cunha Lobo Souto; OLIVEIRA, Naira Vilas Boas Vidal. Intoxicação medicamentosa infantil: um estudo das causas e ações preventivas possíveis. Revista Brasileira de Farmácia, Rio de Janeiro, v. 93, n. 4, p. 422-30, 2012.

MASTROIANNI, Patricia de Carvalho; LUCCHETTA, Rosa Camila; SARRA, Josiane dos Reis; GALDURÓ, José Carlos Fernandez. Estoque doméstico e uso de medicamentos em uma população cadastrada na estratégia saúde da família. Revista Panamericana de Salud Pública, Washington, v. 29, n. 5, p. 358-64, 2011.

MARIANI, Danyelle Cristine; OLIVEIRA, Eliton da Silva. Farmácia caseira e o descarte de medicamentos de moradores da cidade de Itapira-SP. FOCO - Caderno de Estudos e Pesquisas, Mogi Guaçu, n. 9, 2015.

OLIVEIRA, Jéssica Cristina; LIMA, Joice Olívia Medeiros; ZAN, Larissa Bruno; MARCONDES, Gabriela; IHA, Mayara; MARQUES, Luciene Alves. Implantação de postos de coleta para o descarte adequado de medicamentos e subsequente destinação final. InterfacEHS - Saúde, Meio Ambiente e Sustentabilidade, São Paulo, v. 10, n. 1, p. 104-16, 2015. 
Descarte de medicamentos: uma análise da prática de usuários de uma farmácia pública no noroeste do Rio Grande do Sul

PEREIRA, Marlucia Beatriz Lopes; PAULA, Milton Carlos Alves; SANTOS, Nilzalane Lima. Medicamentos: descarte de vencidos e utilização de sobras. Saúde, Santa Maria, v. 44, n. 3, p. 1-8, 2018.

PINTO, Luciano Henrique; CARDOZO, Gilberto; SOARES, Julia Carolina; ERZINGER, Gilmar Sidnei. Toxicidade ambiental de efluentes advindo de diferentes laboratórios de uma farmácia magistral. Revista Ambiente e Água, Joinville, v. 11, n. 4, p. 819$32,2016$.

PINTO, Natália Bitu; LUSTOSA, Jacqueline Pires Gonçalves; FERNANDES, Maria do Carmo de Alustau. O descarte incorreto de fármacos e seus impactos no meio ambiente e na saúde pública. Revista de Pesquisa Interdisciplinar, Cajazeiras, n. 2 (suplementar), p. 563-70, 2017.

RUPPENTHAL, Lísias; PETROVICK, Pedro Ros. Comparação do perfil dos usuários e dos medicamentos dispensados na farmácia popular do Brasil e em drogaria privada em Porto Alegre, Brasil. Latin American Journal of Pharmacy, Buenos Aires, v. 29 , n. 1, p. 9-22, 2010.

SILVA, Jacineide Maria; GERON, Vera Lúcia Matias Gomes. Avaliação de armazenamento de medicamento em domicílio em um bairro de Ariquemes-RO. Revista Científica FAEMA, Ariquemes, v. 9, edição especial, p. 491-99, 2018.

SILVA, Nicole Rodrigues da; ABJAUDE, Samir Antônio Rodrigues; RASCADO, Ricardo Radrighieri. Atitudes de usuários de medicamentos do Sistema Único de Saúde, estudantes de farmácia e farmacêuticos frente ao armazenamento e descarte de medicamentos. Revista de Ciências Farmacêuticas Básica e Aplicada, São Paulo, v. 35, n. 2, p. 319-25, 2014.

SILVA, Thiago José; OLIVEIRA, Vinícius Bednarczuk. Intoxicação medicamentosa infantil no Paraná. Visão Acadêmica, Curitiba, v. 19, n. 1, p. 51-61, 2018. 
